\title{
Ärztliche Professionalität im Zeitalter von Facebook \& Co.
}

\section{Urs Stoffel}

Dr. med., Mitglied des Zentralvorstandes der FMH, Departementsverantwortlicher eHealth - Sicherheitsinfrastruktur und Datenerhebung

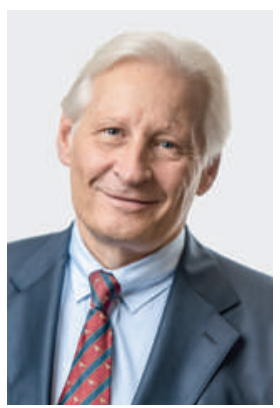

Die Beliebtheit von sozialen Medien hat rasant zugenommen - und diese Entwicklung macht auch vor der Ärzteschaft nicht halt. Internetbasierte Plattformen und Dienste wie Facebook, Google+, Twitter, WhatsApp oder Instagram etc. sind im Alltag kaum mehr wegzudenken. Auch lässt sich private und berufliche Welt zunehmend schwerer auseinanderhalten. Soziale Medien verändern nicht nur die Art und Weise der Kommunikation, sondern ermöglichen darüber hinaus neue Formen der Zusammenarbeit.

Gleichzeitig ergeben sich beim Umgang mit Sozialen Medien für Ärztinnen und Ärzte aber auch spezifische Probleme und Risiken. Internationale Untersuchungen zeigen die häufigsten Fehler von unprofessionellem Verhalten von Medizinalpersonen in Sozialen Medien wie (siehe auch [1]):

- Missachtung von professionellen Kommunikationsund Verhaltensregeln,

- Verletzung der Privatsphäre von Patientinnen und Patienten oder unangebrachte Kontakte zu diesen,

- Offenbarung von vertraulichen Informationen,

- medizinische Empfehlung und Verordnungen von Medikamenten sowie

- ungenaue oder falsche Darstellung von tatsächlichen Qualifikationen oder medizinischen Sachverhalten.

\section{Soziale Medien verändern nicht nur die Kommunikation, sondern ermöglichen neue Formen der Zusammenarbeit.}

Aufgrund der Zunahme von Vorfällen mit disziplinarischen oder juristischen Konsequenzen wird die Notwendigkeit von spezifischen Empfehlungen für Gesundheitsfachpersonen betont.

Im ärztlichen Berufsumfeld stellen sich verschiedene Fragen nach den Grenzen der neuen Möglichkeiten zur Kommunikation und Kollaboration über Soziale Medien: Sollen Ärzte Freundschaftsanfragen von Patienten auf Facebook annehmen? Dürfen Patienten über Soziale Medien beraten oder Empfehlungen zur Behandlung und $\mathrm{zu}$ Medikamenten abgegeben werden? Ist die Übermittlung von vertraulichen Patientendaten über
Soziale Netzwerke zulässig? Was ist zu beachten, wenn in Sozialen Netzwerken oder Online-Communities Fallbesprechungen durchgeführt oder Kommentare über Kollegen abgegeben werden? Beispielsweise ist zu beachten, dass auch im Rahmen eines vermeintlich privaten Auftritts gemachte Aussagen zu Gesundheitsfragen als Aussagen eines Arztes und nicht einer Privatperson gewertet werden können.

\section{Die Empfehlungen der FMH dienen Ärztinnen} und Ärzten als Hilfestellung und Orientierung im Umgang mit Sozialen Medien.

Grundsätzlich gelten die hohen Anforderungen an das professionelle und ethische Verhalten von Ärzten, Medizinstudentinnen und Gesundheitsfachpersonen auch im «öffentlichen Raum» der Sozialen Medien. Negative Schlagzeilen liessen die Frage aufkommen, ob die Standesordnung hinsichtlich Sozialer Medien erweitert werden muss. Die FMH kam jedoch zum Schluss, dass die relevanten Bereiche mit der bestehenden Standesordnung ausreichend geregelt sind. Allerdings muss die Standesordnung im Umfeld neuer Medien richtig interpretiert und umgesetzt werden. Deshalb hat die FMH Empfehlungen zum Umgang mit Sozialen Medien für Ärztinnen und Ärzte ausgearbeitet.

Diese Empfehlungen liegen nun vor und sind auf www. fmh.ch $\rightarrow$ Politik \& Themen $\rightarrow$ eHealth abrufbar. Sie wurden vom Zentralvorstand und der Delegiertenversammlung begrüsst und verabschiedet. Ihnen ging eine umfangreiche Literaturrecherche voraus und sie wurden in der FMH-Arbeitsgruppe eHealth diskutiert. Eine Herausforderung bestand in der Formulierung der Empfehlungen: Sie sollten keine "Verbotsliste» sein, sondern eine Hilfestellung und Orientierung darstellen. Sie müssen konkret und im Alltag praktikabel sein. Gleichzeitig dürfen sie für den Arzt keine unnötigen Hürden für den Eintritt in die elektronische Welt aufbauen. In diesem Sinne sollen die Empfehlungen der FMH für Ärztinnen und Ärzte zum Umgang mit Sozialen Medien auf Risiken und Gefahren aufmerksam machen. 